\title{
Allegric Rhinitis: Pearls of Wisdom
}

\author{
Debashis Acharya* \\ Consultant ENT, Primary Health Care Corporation, Qatar
}

Submission: September 24, 2019; Published: October 17, 2019

*Corresponding author: Debashis Acharya, Consultant ENT, Primary Health Care Corporation, Qatar

\section{Abstract}

\section{Statement of the Problem}

This provides an overview of Allergic Rhinitis and its management. It is very useful for students of Rhinology and clinicians managing this disease. It introduces them to a systematic approach of assessing allergic rhinitis patients which is very commonly found in most populations and causes considerably morbidity. Allergy per se is a very difficult subject to master and it is with great perseverance one can treat patients suffering from this condition. The cornerstone of managing a patient of allergic rhinitis is first and foremost obtaining a good history. This is to be followed by a thorough examination and investigations. The general practitioner is the first expert to be involved in management of allergic rhinitis patient followed by specialists otorhinolaryngologists, and finally by allied healthcare personnel. Inflammation of nose and paranasal sinuses are characterized by two or more symptoms-namely, either nasal blockage / obstruction / congestion or nasal discharge. Associated symptoms include facial pain / pressure and either reduction or loss of smell.

Certain diagnostic endoscopic signs of nasal polyps and or mucopurulent discharge and or mucosal oedema in the middle meatus and or CT changes of mucosa within the ostoemeatal complex, and or sinuses are seen. Definitions, aetiologies, clinical presentations, diagnosis / prognosis and management of allergic rhinitis is dealt with. Common allergens causing the disease are mentioned, pathophysiology and classification of allergic rhinitis is discussed in detail. Different types of allergen testing are highlighted along with their specific role and uniqueness. Principles of immunotherapy in treatment of allergic rhinitis are discussed here. Health effects of allergic rhinitis along with its impact on physical quality of life is mentioned. The basic idea of this presentation is to improve diagnostic accuracy by promoting appropriate use of ancillary tests like nasoendoscopy, allergy testing, computed tomography etc. and reduce inappropriate antibiotic use. The basic treatment plan of allergic rhinitis is according to the severity and duration. It consists of allergen avoidance, pharmacotherapy, allergen immunotherapy and surgery which has limited role.

Keywords: Allergy; Rhinitis; Pollens; Molds; Insects; Penicillium; Cladosporium; Hypersensitivity ; Histamine; Hay fever; Rose fever; Transverse Nasal Crease; Rhinorrhoea; Allergic salute; Allergic Shiners (Dennie -Morgan Lines); Cobblestone appearance of Oropharynx; Scratch Test; Intradermal Test; Patch Test; Rhinomanometry; Antihistaminics; Immunotherapy; Topical Nasal Steroids; Cochrane; Mast cell stabilizer

Abbreviations: IgA: Immunoglobulin A; IgE: Immunoglobulin E; AR: Allergic Rhinitis; NAR: Non-Allergic Rhinitis; ARIA: Allergic Rhinitis \& its Impact on Asthma; >: Greater than; <: Less than; T.M.: Tympanic membrane; NPT: Nasal Provocation Test; n NO: Nitrogen in Nitric Oxide; PNS: Para Nasal Sinuses; OM: Occipito Mental; CECT: Contrast Enhanced Computerized Scan; L.A.: Local Anaesthesia; G.A.: General Anaesthesia; PQLI: Physical Quality of Life Index; WAO: World Allergy Organization; SCIT: Subcutaneous immunotherapy; SLIT: Sublingual immunotherapy; AIT: Allergic Immunotherapy; e-Health: Electronic Health; DBPC: Double Blind Placebo Controlled; RCT: Randomized Controlled Trial; FDA: Food \& Drug Administration federal agency in USA; SMD: Submucous Diathermy; I.T.: Inferior turbinate; FESS: Functional Endoscopic Sinus Surgery; OMC: Osteo-meatal Complex

\section{Introduction}

Rhinitis is a common presentation in E.N.T. clinics across the globe \& allergy compounded with it causes even more difficult to treat for the clinician. This article is useful and handy for students and clinicians managing Allergy\& Rhinitis. There is something in this for everyone - General Practitioners, Otorhinolaryngologists, Allergologists, Rhinologists and Allied Healthcare personnel. Special computer based newer modalities of investigations are highlighted in this which helps in assessing the nasal function of the affected patient. It's very common to sometimes feel like sneezing \& have running nose but please see a doctor if the feeling persists and do take care of yourself. Allergic Rhinitis is made so easy to comprehend [1].

Nasal function includes temperature regulation, olfaction, humidification, filtration and protection. Nasal lining contains secretion of IgA, proteins and enzymes. Nasal cilia propel the matter towards the natural ostia at frequency of 10-15 beats/ 
min. Mucous moves at a rate of $2.5-7.5 \mathrm{ml}$. per min. Rhinitis is the presentation of two or more nasal symptoms for more than one a day namely Nasal congestion/obstruction, Rhinorrhea,
Sneezing, Itching, Impairment of smell. Rhinitis occurs most commonly as Allergic Rhinitis. Non-infectious rhinitis has been classified as either Allergic or Non-Allergic Rhinitis (Figure 1).

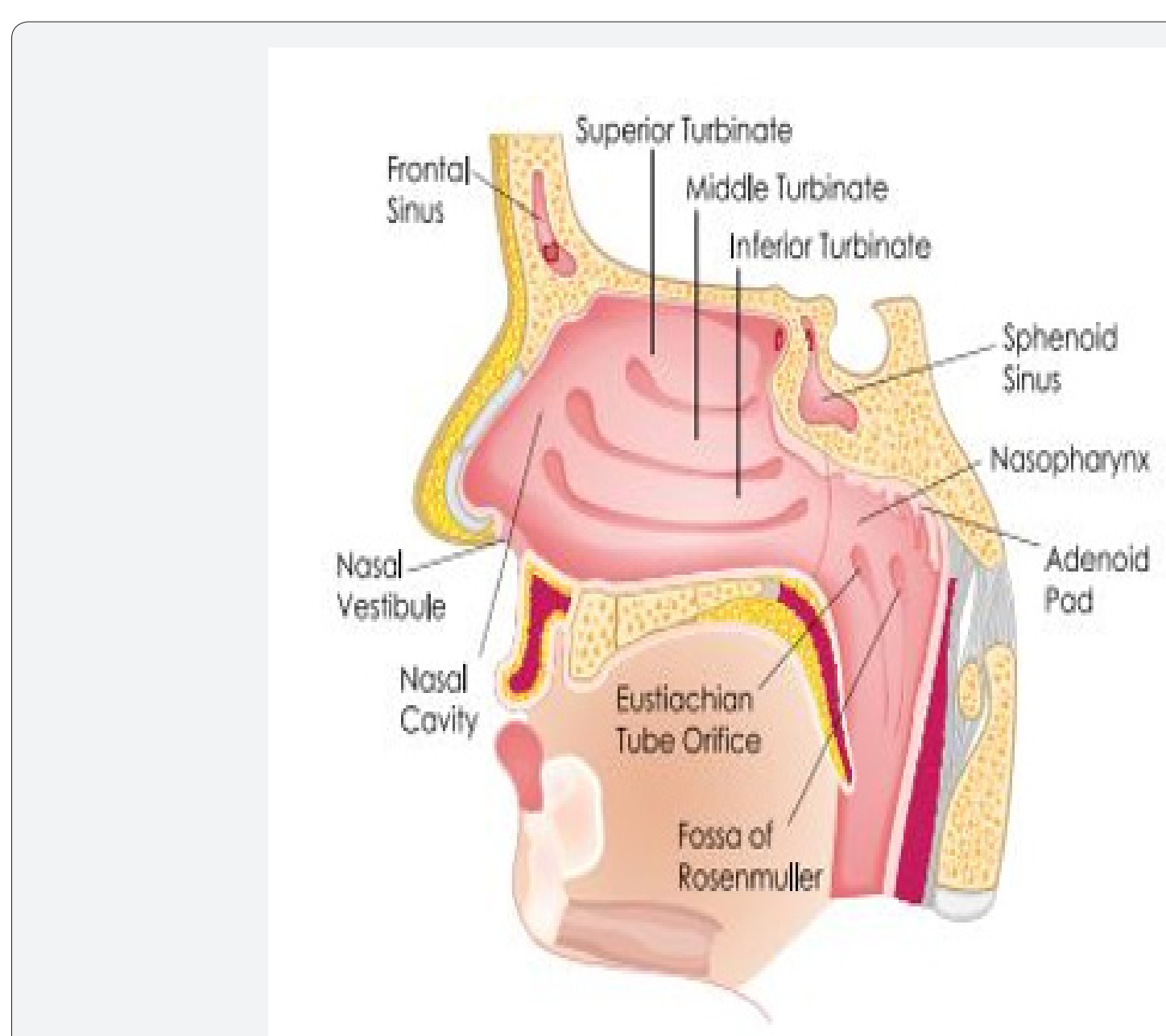

Figure 1: Nasal function.

Allergic Rhinitis affects 15-30 \% of population with a wide geographic variance. It is more common in children \& adolescents. 50\% of all rhinitis in E.N.T. Clinics is Allergic Rhinitis. Allergic Rhinitis is defined as immunologic nasal response, primarily mediated by IgE. Non-Allergic Rhinitis is defined as rhinitis symptoms in the absence of identifiable allergy, structural abnormality or sinus disease. So, Allergic Rhinitis is an inflammation of the nasal mucosa, caused by allergen. It is the most common Atopic allergic reaction [2].

\section{Aetiology}

Aetiology is classified as Precipitating factors and Predisposing factors. Precipitating factors are classified into aerobiological flora and nasal physiology. Aerobiological flora are Allergens present in the environment, House dust \& dust mites, Feathers, Tobacco smoke, Industrial Chemicals and Animal dander. Nasal physiology are Disturbances in normal nasal cycle.
Predisposing factors are classified into genetic, endocrine, psychological, focal sensitivity tests, infections, physical, age \& sex, IgA deficiency and common allergens. Genetic factors indicate towards Multiple gene interactions are responsible for allergic phenotype. Chromosomes 5,6,11,12 \& 14 control inflammatory process in atopy. $50 \%$ of AR pts. Have positive family history [3]. Endocrine factors are Puberty, Pregnancy / Postpartum stages and Menopause.

Infections such as Fungal. Physical factors are Degree of pollution of air, Humidity \& Temperature differences, Temperature changes. Common allergens such as pollens (Spring tree pollens (Maple / Alder / Birch), Summer grass pollen (Blue grass, Sheep sorrel etc.), Autumn Weed pollen (Ragweed)), molds) Penicillium, Cladosporium etc.), INSECTS (Cockroaches, Houseflies, Fleas, Bedbugs) (Figure 2). 

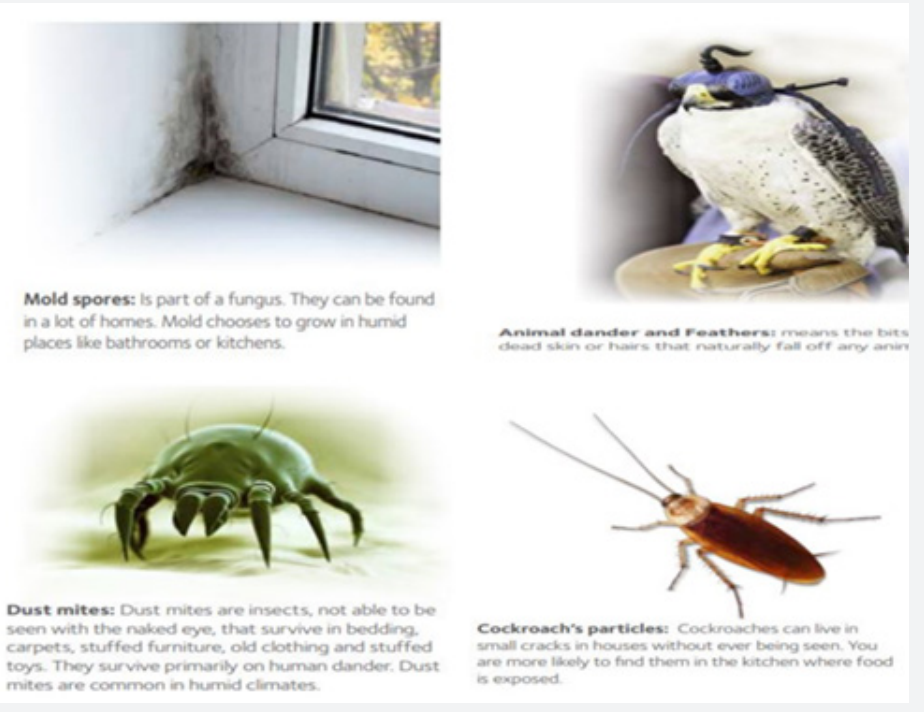

Figure 2: Insects.

\section{Pathophysiology}

Immunoglobulin IgE mediated type 1 hypersensitivity response to an antigen (allergen) in a genetically susceptible person. IgE is produced from plasma cells \& the process is regulated by T-Suppressor lymphocytes or T-helper cells. IgE has affinity for mast cells \& basophils and gets fixed to the surface of mast cells by its Fc end. Type 1 Hypersensitivity causes local vasodilation \& increased capillary permeability. There is edema of the submucosal tissue by allergic fluid followed by infiltration by eosinophils and plasma cells leading to vascular dilatation which causes engorgement of the inferior turbinates and there is increased activity of sero-mucinous glands. Histamine exerts its pharmacologic effect on smooth muscle, vascular endothelium \& mucous glands. Number of IgE molecules has been estimated as 5300 to 27,000 in non-allergic subject \& 15,000 to 41,000 in allergic subjects. Hypersensitivity of the host depends on antigen dose, frequency of exposure, genetic make-up, and hormone activity of the body [4].

\section{Classification}

Allergic Rhinitis is currently classified into intermittent and persistent. In intermittent AR the symptoms are present less than 4 days per week and less than 4 weeks per year. In persistent AR the symptoms are present for greater than 4 days per week and for greater than 4 weeks per year. The severity of AR is classified into mild and moderate to severe. Mild AR doesn't interfere with daily activities or doesn't produce any troublesome symptoms. Moderate to severe AR interferes at least with one of the factors such as impaired sleep, hampered daily activities / work, school / sick absenteeism, also produces troublesome symptoms.

AR is formerly classified into seasonal and perennial based on the allergens. Seasonal Hay Fever, misnomer- no hay / no fever. Summer Cold caused by viruses causing URTI. Rose Fever seen usually in Indian Subcontinent (colourful / fragrant flowering plants). Perennial Allergens present throughout the year [5].

\section{Signs and Symptoms of AR}

Symptoms of AR are sneezing, itching of eyes, ears \& palate, rhinorrhoea, postnasal drip, congestion, anosmia, headache, otalgia, epiphora, red eyes, swollen eyes, fatigue, drowsiness and malaise.

\section{Physical Examination}

During Physical examination we check for Transverse Nasal Crease (Horizontal crease across the lower half of the bridge of nose), Rhinorrhoea (Thin watery secretions from nose). Associated features with AR are Injected \& swelling of palpebral conjunctiva with excess tearing, Retracted T.M.'s, Overbite, Periorbital oedema (Figures 3-6).

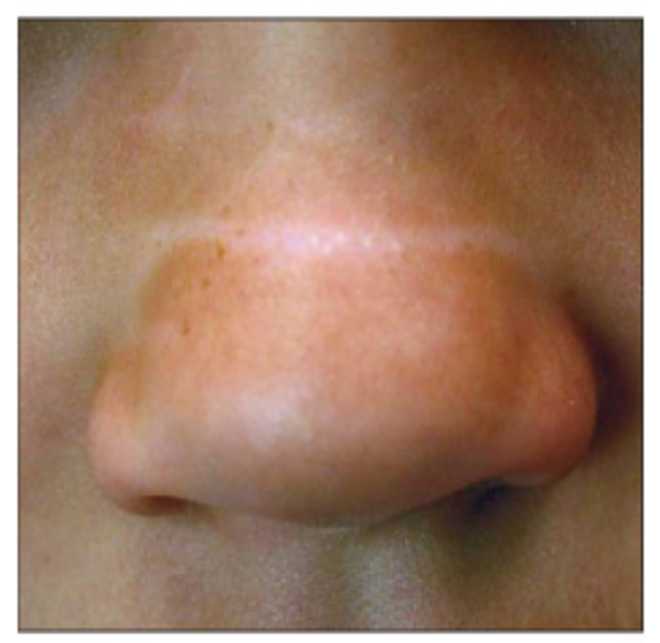

Figure 3: Transverse Nasal Crease. 


\section{Global Journal of Otolaryngology}

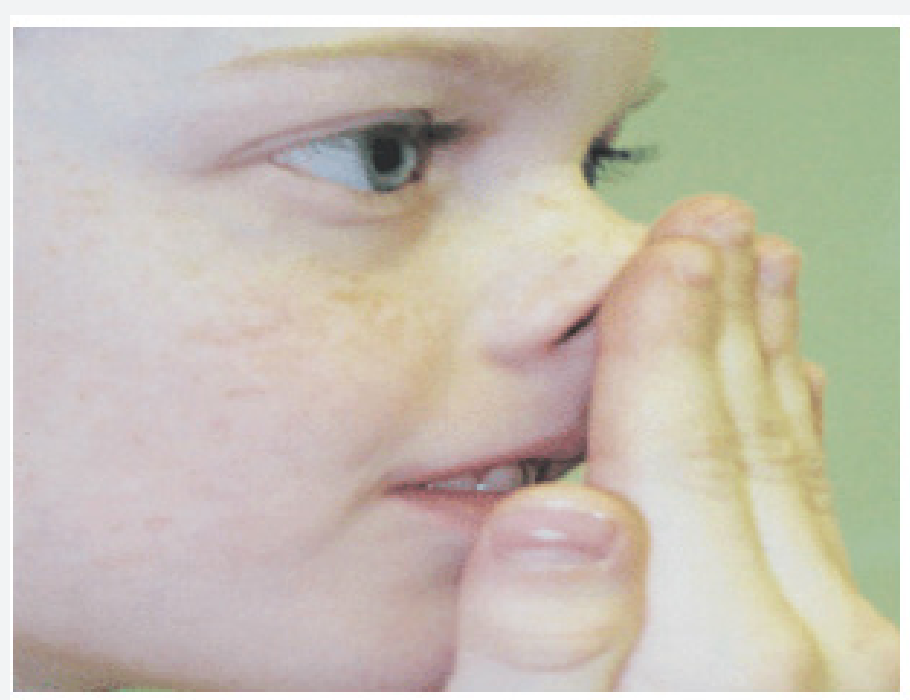

Figure 4: Allergic salute.

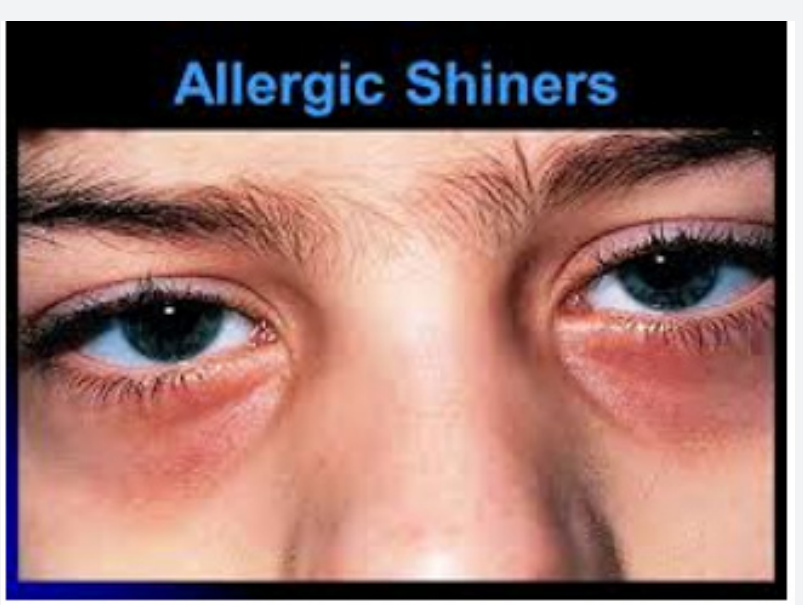

Figure 5: Allergic shiners (Dennie-Morgan lines).

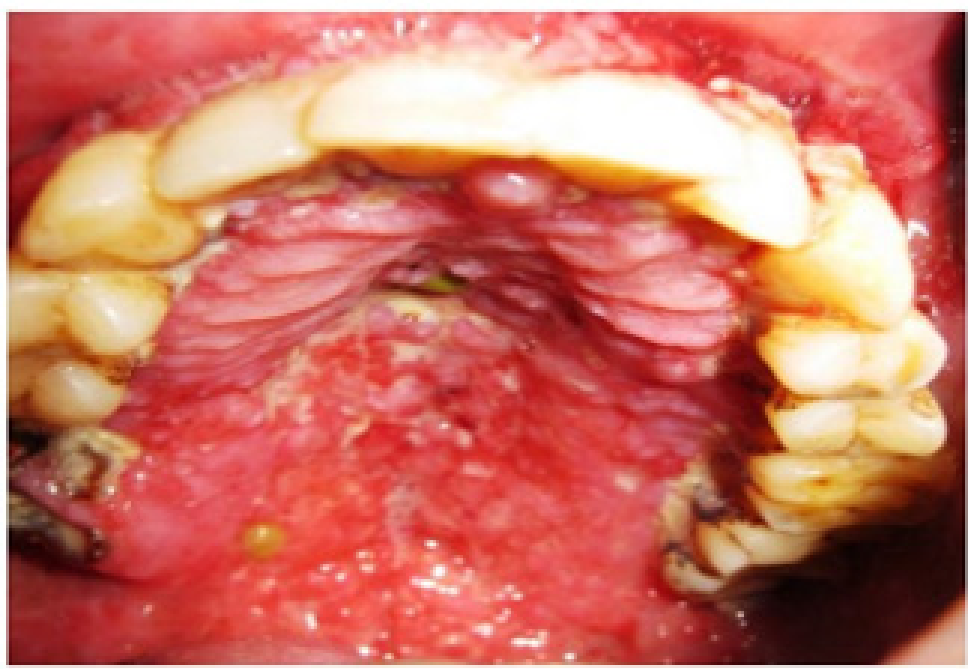

Figure 6: Cobblestone appearance of oropharynx. 


\section{Global Journal of Otolaryngology}

\section{Investigations}

Skin prick tests---gold standard. This is also known as Scratch Test / Intradermal Test. Controlled amounts of allergen \& control substances are introduced into the skin----this procedure is convenient, safe \& widely accepted. Goal of the investigation is the detection of immediate allergic response caused by release of mast cells or basophil IgE specific mediators----Wheal / Flare after 15 mins. More investigations are such as RAST-RadioAllergo-Sorbent Test for specific IgE estimation. PRIST-Plasma
Reactive Immuno-Sorbent Test for specific IgE estimation. SET Test-Skin End-Point Titration Test Latest skin test for allergy \& is more reliable (Figure 7). Less common tests are total serum IgE, total blood eosinophil count, nasal smears may show increased eosinophilic level, PATCH TEST is used to determine delayed type hypersensitivity \& the allergen is placed in the skin for 48 hrs. The area of reaction is noted and if any allergens present are identified. This is more useful in skin problems \& food allergy [6].

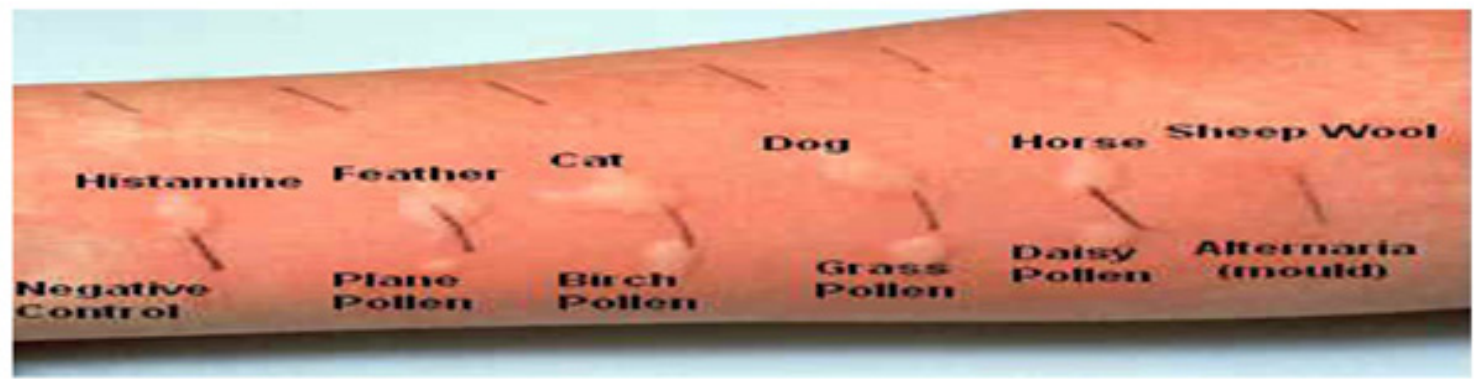

Figure 7: Scratch Test.

\section{Nasal provocation tests (NPT)}

The potential allergen is sprayed into the nose $\&$ the number of SNEEZES counted or any change in Rhinomanometry is noted. Very time-consuming test as each allergen takes 20 mins. To test in order to allow the nose to return to normal after the challenge. It is useful for rare \& occupational allergens. Contraindications of NPT are Pregnancy / < 5yrs. age / Recent nasal surgery < 8 wks. / Uncontrolled asthma, Nasal / systemic corticosteroids should be avoided for 1 wk. \& Antihistaminics for 72 hrs (Figures 8 \& 9).

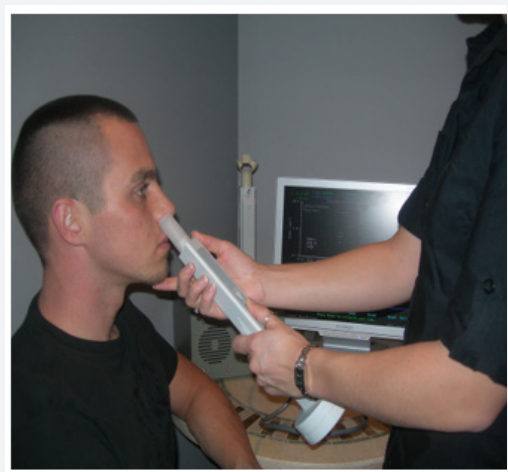

Figure 8: Rhinometer.

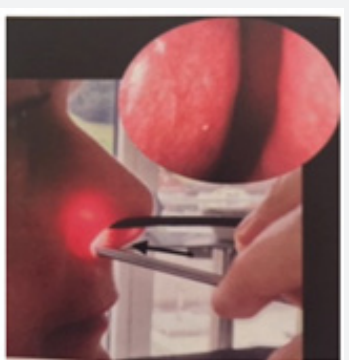

Figure 9: : Spraying Device for NPT. 
New Diagnostic Methods

\section{Exhaled Nitric Oxide (E No)}

Like e NO in asthma, n NO is a non-invasive marker. Potentially suitable to monitor upper airway inflammation following allergen-induced late response. In AR pts., increased levels of $\mathrm{n}$ NO have been measured. However, the applicability of $\mathrm{n} \mathrm{NO}$ as a marker of upper airway inflammation awaits validation. Exhaled nitric oxide (e NO) is currently the most reliable marker of rhino-bronchial inflammation, but its routine assessment is difficult as the test is available only in highly specialized centers [7].

\section{Other Investigations}

X-RAY PNS---Water's ( OM view). CECT of PNS....2mm. Coronal cuts are preferred nasoendoscopy under L.A. / G.A. Hopkins rod ( $\left.0^{\circ} / 30^{\circ} / 70^{\circ}\right)$. Evaluate the individual for asthma. At some centers FAST-Fluro Allergo Sorbent Test is done.

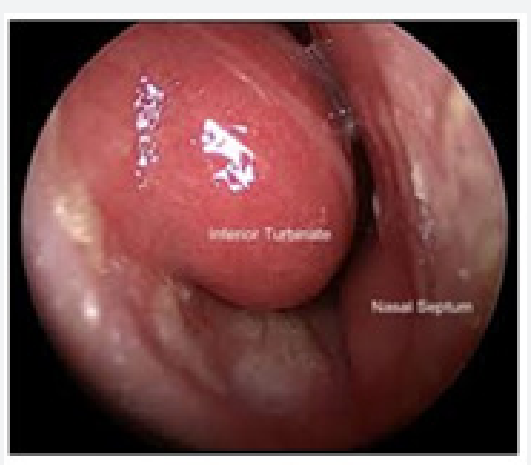

Figure 10: Chronic Nasal.

\section{Complications of AR}

Complications of AR are allergic asthma, chronic otitis media, hearing loss, chronic nasal obstruction, sinusitis, orthodontic malocclusion in children (Figure 10).

\section{Prognosis}

Treatment is available \& pts. remain asymptomatic only until re-exposure to allergic antigen. There is no evidence of mortality from the disease but there is very high morbidity. PQLI is affected. Seasonal allergic symptoms improve as patients age.

\section{Management of AR}

Management of Allergic Rhinitis includes Allergen avoidance \& environmental control measures, Medical / pharmacologic treatment, Immunotherapy and Surgery. Choice of treatment will depend on efficacy, safety, cost-effectiveness, patient preferences, combination, objectives of treatment, likely adherence to recommendations, severity \& control of disease and presence of co-morbidities. Practical allergen avoidance tips given by WAO for public education purposes are as follows (Figure 11).
- House dustmite allergen reduction : Aims to reduce the amourt of mite allergens in the home - Wash beding regularly (every 1.2 weeks) at $55-60^{\prime} \mathrm{C}$, If possible, to kil mites.

- Ensure sufficient ventiation of residence to decrease humidity and avoid damp housing conditions.

- Use a damp cloth whon dusting and cleaning surfaces.

- Avoid using carpets so that the floor can be wiped clean.

- Remove / reduce curtains and soft furnishings is the bedroom.

Remove soft toys from the bedroom.

\section{Pet allergen avoidance :} Reduces the amount of pet allergen indioors.

- If posslole keep pets actidoors.

- Exclude pets from bedrooms.

- Vecum carpets and mattresses regularly, If vacuum cleaners are avalable.

- Change clothes before going to schooV workif you have attended your herseicat doe.

Figure 11: Few practical allergen avoidances tips.
- Pollen avoidance :

Provides mechanical bariers to polen conbet

- Keep windows closed at peak polien imes, 0.9. in the evening when arborne polens descend to lower attudes.

- Wear glasses or sunglasses to prevent entering the eyes.

- Consider wearing a mark over nose and mouth to prevent inhalation of pollens at peaktme.

- Donotcut grass yourselt.

- Keep windows closed when the grass the been cut.

\section{In the School environment :}

- Ensure that parents, teachers, fellow students and school administrators are aware of the necessity to provide a safe envirorment for children at risk for anaphytais to foods or insectstings. 


\section{Pharmacotherapy}

Oral antihistaminic are 1ST GEN.:- Chlorpheniramine maleate/Diphenhydramine/Clementine, 2ND GEN.:- Loratadine/ Terfenadine/Acrivastine, 3rd GEN.:- Fexofenadine/Cetrizine. Topical application of Azelastine. NEWER - Desloratadine / Levocetrizine.

\section{Acute Phase Medications}

Antihistaminics are effective in blocking histaminic effects. (Runny nose/Watery eyes). Side Effects of antihistaminics are Sedation, Dry mouth, Nausea, Dizziness, Blurred vision, Nervousness. Non-sedating antihistaminics (Cetirizine/ Loratadine) has fewer side effects. Fexofenadine is more effective (has a lower risk of cardiac arrythmias). Decongestants will Shrink mucous membranes by vasoconstriction. These are available OTC / in combination with antihistaminic, analgesics \& anti cholinergic. Anticholinergic Agents Inhibit mucous secretions which acts as drying agents. Topical Eye Preparations reduces inflammation / relieves burning and itching (Figure 12).

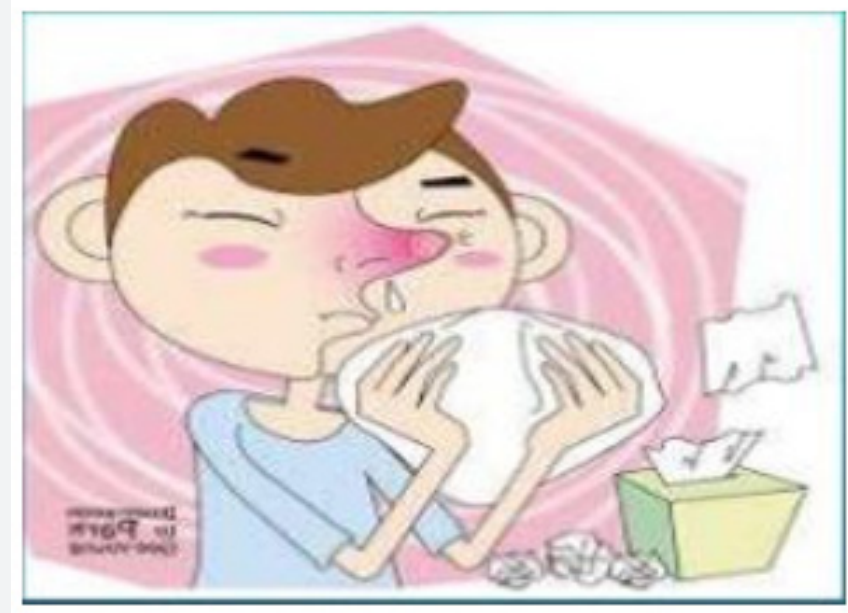

Figure 12: Blocking histaminic effects.

\section{Preventive Therapy}

Intranasal Corticosteroids Reduces inflammation of mucosa, prevents mediator release, can be used safely daily, can be given systemically as a short course during a disabling attack. Intranasal Cromolyn Sodium Mast cell stabilizer Prevents release of chemical mediators. Oral Mast Cell Stabilizer, Ophthalmic solution cromolyn [8].

Topical nasal steroids are dexamethasone, beclomethasone dipropionate, triamcinolone acetonide, flunisolide, budesonide, fluticasone propionate, mometasone furoate and ciclesonide. Leukotriene Receptor Antagonists (Anti Leukotriene Agents) are Monteleukast( Singular)/Zafirleukast(Accolate) \& Pranleukast. These drugs reduce inflammation, oedema \& mucous secretions of Allergic Rhinitis. ZILEUTON (5-Lipoxygenase inhibitor) is a similar drug \& is used in many parts of the world [9].

\section{Immunotherapy (Ait)}

SCIT is effective in seasonal polleniosis \& mite allergy. SLIT is Effective \& safe alternative, best in seasonal AR. COCHRANE reviews shows that both are equally effective \& the patient is in equipoise. These are more effective in adult pts. 3yrs. Of treatment with both SCIT \& SLIT has been shown to provide long term clinical benefits for at least 2 yrs. after their discontinuation. The choice of therapy depends on grounds of convenience, availability of resources \& personal preferences. SCIT requires administration in a specialist clinic whereas SLIT can be selfadministered.

\section{Current Concepts \& Future Needs}

Although AIT is considered a safe \& effective treatment for AR, however, its clinical effect is still contested by many due to: Heterogenicity in clinical trial designs, Study populations, Therapeutic formulations, Efficacy criteria. There is ample scope for physicians, patient organizations, companies \& regulators to improve clinical trials in AIT and, to provide patients with better treatments. Inclusion of allergic pts. with relevant diseases(s) in AIT trials.

Exclusion of polyallergic pts. (with clinically relevant, overlapping allergen exposures) in AIT trials. Clinically defined responders in AIT trials. Allergen exposure chambers in AIT trials. Differences in regional \& seasonal exposures. Adaptive trial designs should be discussed with regulatory bodies. Patient-to-patient differences in treatment adherence \& allergen exposure. (Use of "e-health" is recommended). The placebo effect in AIT is to be considered. Ethical \& technical aspects of DBPC / RCT's, especially in paediatric populations. The importance of safety reporting. (WAO guidelines for reporting systemic \& local adverse events should be applied) [10]. 


\section{Global Journal of Otolaryngology}

\section{Omalizumab for Treatment of AR}

This is a new treatment strategy for allergic rhinitis DBPC study of RAGWEED immunotherapy7 is done. Prohibitive high cost. It is not FDA approved for this indication. Periodic use is justified in treatment of resistant patients especially those with seasonal disease. This drug acts by removal of circulating free IgE by the recombinant humanized monoclonal anti IgE antibody (Figure 13).
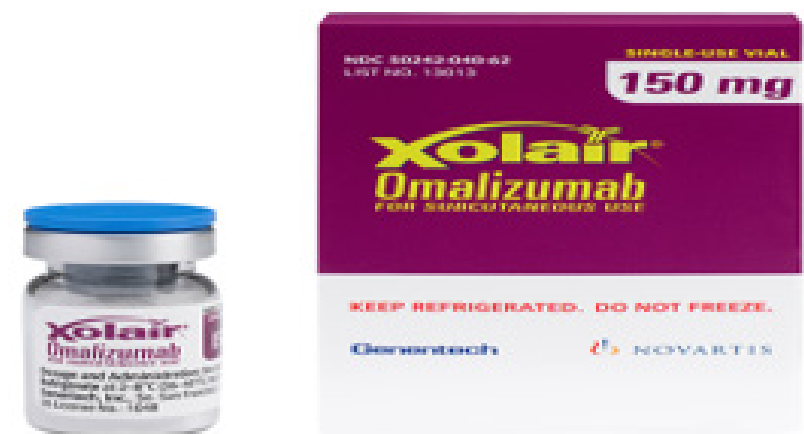

Newe nermoereared. Do nor rmescr.

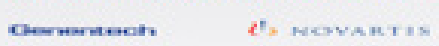

Figure 13: Drugs for AR.

\section{Surgical Treatment}

Surgical treatment has limited use, SMD of I.T. reduces the size of boggy turbinates. Septoplasty is done for the Correction of septal deviation. FESS is done for the Clearance of sinuses and OMC If indicated. VIDIAN NEURECTOMY is done in certain selected cases, Surprising relief for stuffy nose: Sex.

According to Dr. Michael Benninger, Otolaryngologist, Chairman of Cleveland Clinic's Head \& Neck Institute, in a study done in May 2018 has some surprising findings. The potential effect of sexual activity works the same for man \& women. Swollen tissues in nose block the passages in AR creating congestion \& making it harder to breathe. During arousal, the Sympathetic NS gets into play, adrenaline levels go up \& blood vessels constrict. Less blood flow to the nose means less inflammation, so the nose opens up \& one can breathe more easily. Lying on back position for both men and women, one cannot experience the same level of congestion relief due to effects of gravity (Figure 14).

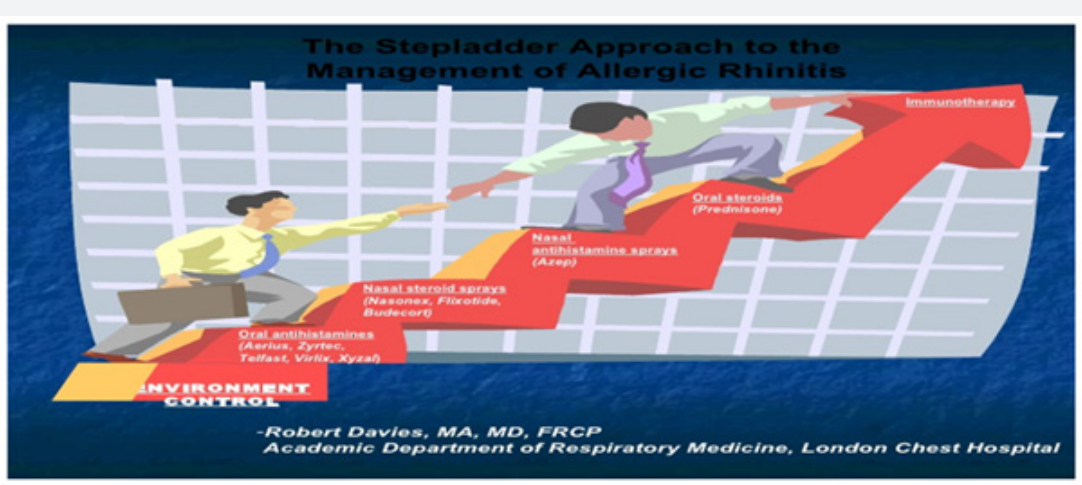

Figure 14: Stepladder approach in treatment of AR.

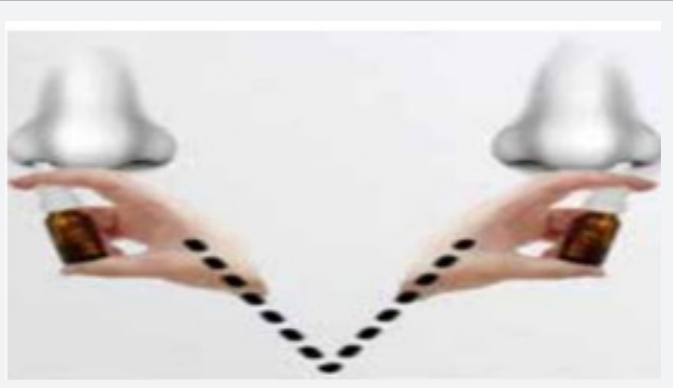

Figure 15: Nasal Sprays. 


\section{Allergic Rhinitis Guide: Frequently Asked Questions (FAQ'S)}

i. What is common medication mistake that people make?

ii. How long should one stay on allergy treatment?

iii. How can one differentiate between common cold \& AR?

iv. What are the side effects of allergy medications?

v. How to use nasal sprays? (Figure 15)

vi. Are the steroids in nasal sprays safe?

vii. What is the difference between the nasal medications \& oral medications?

viii. How quickly can one expect to get relief from allergy treatment?

ix. Taking corticosteroids inhalers to control asthma symptoms, can one also take allergy medications?

x. What are the types of allergy tests?........IgE skin test / Intradermal test /Specific IgE in blood.

\section{References}

1. Oliver Hausmann, Markus H F Pfister (2015) Recent advances in Otolaryngology Head \& Neck Surgery 4: 14.

2. Color Aids: E.N.T. (1988) Nicholas D, Stafford \& Robin Young.

3. S K De's (2017) Fundamentals of Ear, Nose \& Throat \& Head-Neck Surgery 2(6): 202-203.

4. Logan Turner's Diseases of the Nose Throat and Ear (2017) 10(Ed) 1.8: 52.

5. Ian S Mackay, T R, BullScott Brown 's Otolaryngology: 5(Ed), Rhinology, 7.

6. Robert Davis, Academic dept. Of Respiratory Medicine, London Chest Hospital.

7. Mehdi Adeli, SrCons. Allergy \& Immunology, AIAP- Paed. Dept., HMC, Doha, Qatar.

8. Sarita Pandey: Allergic Rhinitis: SlideShare: LinkedIn.

9. Google Images.

10. O Pfaar, M Alvaro, V Cardona, E Hamelmann, R Mosges, et al. (2018) Clinical trials in allergen immunotherapy: current concepts and future needs. Allergy 73(9): 1775-1783.

\begin{tabular}{l} 
Your next submission with Juniper Publishers \\
will reach you the below assets \\
- Quality Editorial service \\
- Swift Peer Review \\
- Reprints availability \\
- E-prints Service \\
- Manuscript Podcast for convenient understanding \\
- Global attainment for your research \\
- Manuscript accessibility in different formats \\
( Pdf, E-pub, Full Text, Audio) \\
- Unceasing customer service \\
Track the below URL for one-step submission \\
https://juniperpublishers.com/online-submission.php \\
\hline
\end{tabular}

